\title{
The crystal structure of a high affinity RNA stem-loop complexed with the bacteriophage MS2 capsid: Further challenges in the modeling of ligand-RNA interactions
}

\author{
WILF T. HORN, ${ }^{1}$ MÁIRE A. CONVERY, ${ }^{1,3}$ NICOLA J. STONEHOUSE, ${ }^{1}$ CHRIS J. ADAMS, ${ }^{1}$ LARS LILJAS, ${ }^{2}$ \\ SIMON E.V. PHILLIPS, ${ }^{1}$ and PETER G. STOCKLEY ${ }^{1}$ \\ ${ }^{1}$ Astbury Centre for Structural Molecular Biology, University of Leeds, Leeds LS2 9JT, United Kingdom \\ ${ }^{2}$ Department of Cell and Molecular Biology, Uppsala University, SE-751 24 Uppsala, Sweden
}

\begin{abstract}
We have determined the structure to $2.8 \AA$ of an RNA aptamer (F5), containing 2'-deoxy-2-aminopurine (2AP) at the -10 position, complexed with MS2 coat protein by soaking the RNA into precrystallised MS2 capsids. The -10 position of the RNA is an important determinant of binding affinity for coat protein. Adenine at this position in other RNA stem-loops makes three hydrogen bonds to protein functional groups. Substituting 2AP for the $\mathbf{- 1 0}$ adenine in the F5 aptamer yields an RNA with the highest yet reported affinity for coat protein. The refined X-ray structure shows that the 2AP base makes an additional hydrogen bond to the protein compared to adenine that is presumably the principal origin of the increased affinity. There are also slight changes in phosphate backbone positions compared to unmodified F5 that probably also contribute to affinity. Such phosphate movements are common in structures of RNAs bound to the MS2 $T=3$ protein shell and highlight problems for de novo design of RNA binding ligands.
\end{abstract}

Keywords: bacteriophage MS2; crystal structure; RNA-protein interactions; RNA stem-loop

\section{INTRODUCTION}

RNA-protein interactions play crucial roles in many areas of gene expression and protein synthesis. In addition, RNAprotein complexes are the targets for vital clinical drugs such as antibiotics. Major drug development programs are aimed at disrupting RNA-protein complexation. Such studies are underpinned by our understanding of the molecular basis of protein-RNA interactions. The RNA bacteriophages of Escherichia coli have long been used as models in which such details can be probed easily.

Bacteriophage MS2 has a single-stranded RNA genome that is encapsidated in a $T=3$ protein shell constructed from three chemically identical but structurally distinct protein conformers $(\mathrm{A}, \mathrm{B}$, and $\mathrm{C})$, that associate to give $\mathrm{AB}$ and CC dimers. The dimers are the basic building blocks of

Reprint requests to: Peter G. Stockley, Astbury Centre for Structural Molecular Biology, University of Leeds, Leeds LS2 9JT, United Kingdom; e-mail: stockley@bmb.leeds.ac.uk; fax: 0113-233-3092.

${ }^{3}$ Present address: Structural and Biophysical Sciences, GlaxoSmithKline Medicines Research Centre, Gunnels Wood Road, Stevenage, Hertfordshire, SG1 2NY, United Kingdom.

Article and publication are at http://www.rnajournal.org/cgi/doi/ 10.1261/rna.7710304. the icosahedral shell. In vivo, a 19-nt RNA stem-loop (TR; Fig. 1) within the viral genome is bound sequence-specifically by a coat protein dimer as the concentration of coat protein rises during infection. The complex formed represses translation of the downstream viral replicase cistron. It also appears to act as the assembly initiation trigger for capsid self-assembly, thus ensuring encapsidation of the cognate genomic RNA (Ling et al. 1970; Witherell et al. 1991; Stockley et al. 1994).

For many years this interaction has been a major paradigm for studying the details of RNA-protein interactions via both structural and biochemical assays (Witherell et al. 1991; Valegård et al. 1994; Stockley et al. 1995; Convery et al. 1998; Rowsell et al. 1998; Parrott et al. 2000; Grahn et al. 2000, 2001). These studies have revealed a consensus RNA sequence that retains all of the features recognized by the coat protein dimer. For high affinity binding, the -4 and -7 positions (numbered with respect to the replicase start codon; Fig. 1) must be adenines, although the bases play different roles in the interaction. The A-4 binds into a shallow pocket on the surface of one subunit in the protein dimer, where it makes a series of intermolecular hydrogen bonds and hydrophobic contacts. Six nucleotides 5' from 


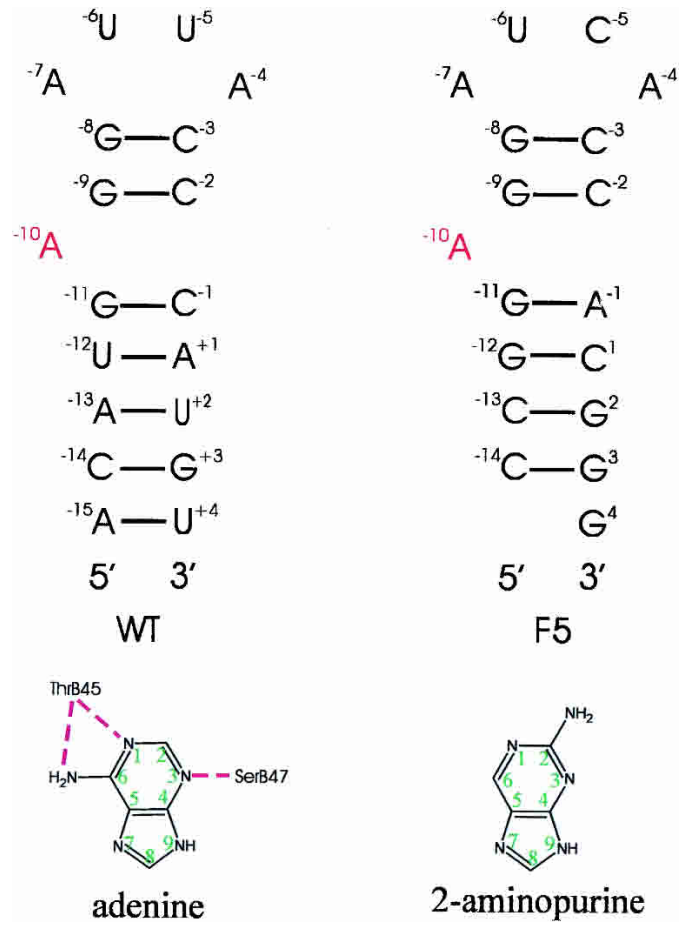

FIGURE 1. (Top) Diagram of the secondary structures of the wildtype MS2 RNA stem-loop (TR) (left) and the F5 RNA aptamer (right) (Rowsell et al. 1998). The adenine at the -10 position is shown in red. (Bottom) Structures of adenine showing the hydrogen bond interactions that are made with coat protein at the -10 position (left) and 2-aminopurine (right).

A-4 (i.e., position -10) an unpaired purine should also be present. In unliganded stem-loops this intercalates between the neighboring base pairs (Borer et al. 1995), but in the complex it becomes extruded and binds into a pocket in the other subunit. In contrast, the A-7 does not contact the protein directly; rather it is part of a stacking interaction with the base pair in the stem below it and with one of the pyrimidines $(-5)$ in the loop. The -5 base also stacks on the side chain of Tyr 85 in the protein. High affinity binding also requires the presence of a pyrimidine at -5 . This residue participates in the stacking interaction mentioned above and the $\mathrm{O} 2$ of the ring forms a hydrogen bond to Asn 87. Stem-loops having $\mathrm{C}$ at position -5 exhibit higher affinity than the wild-type U-5 sequence, apparently because the exocyclic 4 -amino group makes a stabilizing contact to the phosphodiester at position -6 (see below).

In part, these rules for the RNA consensus sequence are derived from the analysis of the crystal structures of a large number of RNA stem-loops encompassing both sequence and functional group variations. These were obtained using crystals of the recombinant $T=3$ protein shell prepared from E. coli (Mastico et al. 1993). Such capsids lack specifically bound RNAs and allow short stem-loop oligonucleotides to be soaked into the crystals. These access the RNAbinding sites in the interior of the capsids via pores at the capsid symmetry axes (Valegård et al. 1994, 1997). The protein capsid acts as a scaffold allowing the determination of the details of the RNA-protein interaction via simple difference maps.

Stem-loops that have been studied in this way include three aptamers, F5, F6, and F7, generated by in vitro selection, that differ in their secondary structures compared to the wild-type TR RNA (Convery et al. 1998; Hirao et al. 1998; Rowsell et al. 1998). One of these, F5 (Fig. 1), has an $\sim 20$-fold higher affinity for MS2 coat protein in vitro compared to the wild-type RNA, consistent with the presence of a C-5 substituent. We have studied the solution behavior of the F5 and F6 aptamers substituted with the analog 2'deoxy-2-aminopurine (2AP) at the $-4,-7$, and -10 sites using fluorescence spectroscopy (Parrott et al. 2000). The F5 aptamer, substituted at the -10 position with $2 \mathrm{AP}$ (termed the F5/2AP10 RNA; Fig. 1), has an $\sim 65$-fold higher affinity compared to the TR (Parrott et al. 2000), making it the best ligand yet identified for the protein. Substitution by deoxy adenine at the $-4,-7$, or -10 positions is known to be essentially silent in terms of protein affinity (Stockley et al. 1995), so the difference in affinity must be due to the changes in the purine ring. To understand the molecular basis for this changed affinity we have used the capsid scaffold approach to determine the X-ray crystal structure of the F5/2AP10 RNA complexed with wild-type $T=3$ capsids.

\section{RESULTS AND DISCUSSION}

The F5/2AP10 RNA-protein complex was refined to a resolution of $2.8 \AA$ (Tables 1,2). The high quality of the electron density maps allowed all protein residues to be modeled. The protein adopts the same overall topology seen in intact phage (Golmohammadi et al. 1993). The amino acid side chains of Asn A87 (residue 87 of the A subunit within an $A B$ dimer) and Lys A61, which have each been modeled in two distinct conformations in earlier structures (Valegård et al.

TABLE 1. Statistics from data collection and scaling for the F5/2AP10 complex

\begin{tabular}{lc}
\hline Complex & $\mathrm{F} 5 / 2 \mathrm{AP} 10$ \\
\hline Resolution $(\AA)$ & $30-2.8$ \\
No. reflections & 559,447 \\
No. reflections (unique) & 170,082 \\
No. crystals & 6 \\
$R_{\text {merge }}{ }^{2}$ & $14.6(34.4)$ \\
$l / \sigma l$ (mean) & $2.0(1.6)$ \\
Completeness $(\%)$ & $60.8(23.8)$ \\
\hline
\end{tabular}

Numbers in parentheses correspond to the highest resolution bin $(2.9-2.8 \AA)$.

$$
{ }^{\mathrm{a}} R_{\text {merge }}=\frac{\sum_{h k l} \sum_{i} \mid l_{i}(h k l)-\overline{l(h k l)}}{\sum_{h k l} \sum_{i} l_{i}(h k l)} \times 100
$$


TABLE 2. Refinement statistics for the F5/2AP10 complex

\begin{tabular}{lc}
\hline Complex & F5/2AP10 \\
\hline$R$-factor $^{\mathrm{a}}$ & 18.8 \\
$\mathrm{R}_{\text {free }}$ & 19.6 \\
RMS deviations $^{\mathrm{c}}$ & \\
$\quad$ Protein: bonds $(\AA)$ & 0.006 \\
$\quad$ angles $\left({ }^{\circ}\right)$ & 1.3 \\
Ramachandran plot $(\%)$ & \\
$\quad$ Most favored regions & 87.8 \\
$\quad$ Disallowed regions & 0.3 \\
Average B-factor $\left(\AA^{2}\right)$ & \\
Protein & 30.8 \\
RNA & 53.4 \\
Water molecules & 51.0 \\
RNA occupancies & \\
AB RNA & 0.9 \\
CC RNA & 0.5 \\
Refined model & \\
Protein residues & 387 \\
Nucleotides & 34 \\
Water molecules & 159 \\
\hline
\end{tabular}

Ramachandran plots were calculated using the program PROCHECK (Laskowski et al. 1993).

${ }^{\mathrm{a}} R$-factor $=100 \times \sum \| F_{\text {obs }}|-k| F_{\text {calc }}|| \sum\left|F_{\text {obs }}\right|$.

${ }^{\mathrm{b}} R_{\text {free }}=100 \times\left.\sum\right|_{T}\left|F_{\text {obs }}\right|-k\left|F_{\text {cald }}\right| / \Sigma_{\mathrm{T}}\left|F_{\text {obs }}\right|$ (calculated using a test set of $4 \%$ of reflections).

${ }^{\mathrm{C}}$ Deviation from ideal values.

1997; Rowsell et al. 1998) display a single conformation in the complex. Reasons for this are unclear but are possibly due to the high occupancy of the F5/2AP10 RNA, leading to the side chains of both residues being in their "bound" conformation. Asn A87 makes the hydrogen bond contact with the $\mathrm{C}-5 \mathrm{O} 2$ position of the RNA. The hydrophobic portion of the Lys A61 side chain interacts with the face of A-4. No conformational changes to the side chains of amino acids within the -10 binding pocket, compared to the F5 complex, were observed. These data suggest that the increased affinity is the result of the changes made to the RNA alone.

RNA stem-loops bind to both $\mathrm{AB}$ and $\mathrm{CC}$ dimers within the capsid. Due to the symmetry of the CC dimer, the RNA binds in two mutually exclusive orientations, making definitive interpretation of the protein-RNA interactions at these sites problematic. In contrast, RNA stem-loops bind $\mathrm{AB}$ dimers in a single orientation, allowing an unambiguous interpretation of the electron density at these sites. Although RNA was modeled and refined at both the $\mathrm{AB}$ and CC sites, all the protein-RNA interactions discussed below refer to those observed at the $\mathrm{AB}$ binding site.

$2 F o-F c$ electron density maps clearly show interpretable density for the entire F5/2AP10 stem-loop (Fig. 2) with the exception of the $3^{\prime}$ guanidine, which exhibits weak density, probably because of its lack of a Watson-Crick base-pairing partner (Fig. 1). There is also weak density for the unpaired uracil at the -6 position, but in previous structures density for this residue is variable and does not easily correlate with RNA occupancy or affinity (Valegård et al. 1997; Convery et al. 1998; Rowsell et al. 1998, Grahn et al. 2000, 2001). 2Fo$F c$ maps, calculated with the 2AP base omitted from the F5/2AP10 model, exhibit strong electron density at the site of the 2AP substitution, allowing unambiguous modeling of the conformation of this base (Fig. 3). The tertiary structure of the F5/2AP10 RNA is very similar to that of the F5 aptamer (Rowsell et al. 1998). The root mean square deviation of the 17 common phosphorus atoms of the two structures, calculated with the program LSQMAN (Kleywegt and Jones 1997) is $0.34 \AA$ (Fig. 2). All ribose sugars were modeled in the C3' endo conformation, with the exception of the sugars at the $-4,-5$, and -6 positions, which are C2' endo, as seen in previous complexes (Valegård et al. 1997; Convery et al. 1998).

The most significant changes at the protein-RNA interface occur at the site of the 2AP substitution. Movement of the exocyclic amino group of the base from the $\mathrm{C} 6$ to the $\mathrm{C} 2$ position might have been expected to delete the hydrogen bond from $\mathrm{N} 6$ to the O $\gamma$ of Thr B45 (Fig. 4). However, the orientation of the base is such that this interaction is still made by $\mathrm{N} 2$, and its distance from the $\mathrm{O} \gamma$ is essentially

A
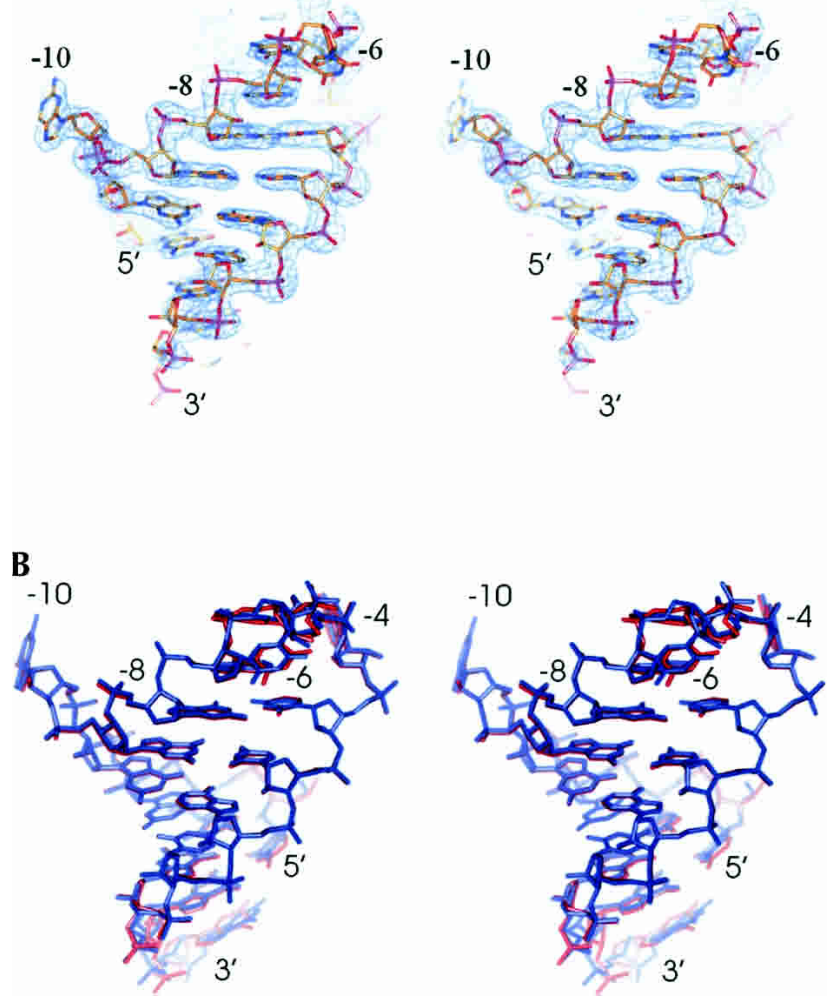

FIGURE 2. Stereo diagrams of the F5/2AP10 RNA at the AB binding site with associated $2 F o-F c$ electron density contoured at 1 RMS $(A)$, superposition of the F5/2AP10 (blue) and the F5 aptamer (red) RNAs showing the overall conformational similarity of the sugar-phosphate backbone $(B)$. Figure created using the program SPOCK. 


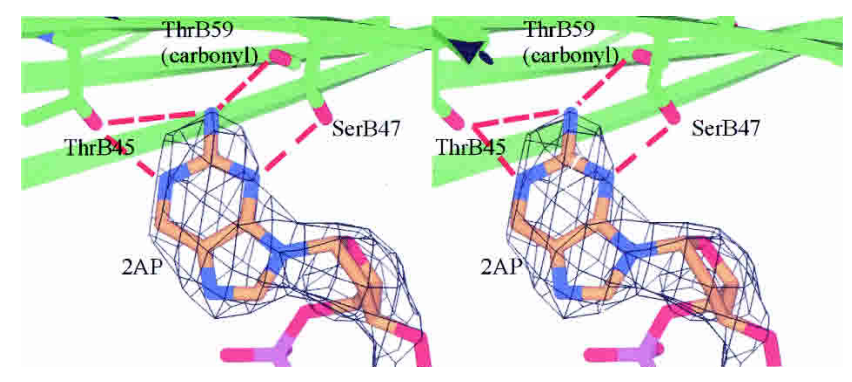

FIGURE 3. Stereo diagram showing $2 F o-F c$ electron density, calculated with the $2 \mathrm{AP}$ base omitted from the F5/2AP10 model, at the site of the 2AP substitution. Density is contoured at 1 RMS and has been overlaid with the $2 \mathrm{AP} 10$ base from the final F5/2AP10 structure. Amino acid residues that form hydrogen bond interactions (dashed red lines) with the 2AP base are represented in stick format. Figure created using the program SPOCK.

unchanged. The N2 amino group also makes an additional hydrogen bond with the backbone carbonyl of Thr B59. This is a novel contact for bases at the -10 position, but is one of the interactions seen at the -4 binding pocket, where the orientation of the adenine is different and N6 can contact both Thr A45 and Thr A59. Hydrogen bond contacts from the $\mathrm{O} \gamma$ of Thr B45 and from the $\mathrm{OH}$ of Ser B47 to the N3 and N1 positions of the ring, respectively, are still made in the F5/2AP10 complex. These observations strongly suggest that it is the additional hydrogen bond from the 2AP exocyclic amino group to Thr B59 that is primarily responsible for the high affinity of this RNA variant (Fig. 4).

The ring plane of the 2AP base is rotated slightly $\left(\sim 8^{\circ}\right)$ within the -10 binding pocket relative to adenine at this position in the F5 complex. This small change in orientation moves the $\mathrm{C} 2$ amino group and Thr B59 closer together and is probably a consequence of forming the new hydrogen bond contact. It also leads to a more favorable geometry for the hydrogen bond between the $\mathrm{C} 2$ amino group and the $\mathrm{O} \gamma$ of Thr B45. Minor changes also occur to the position of the -7 phosphate, bringing it closer to the $\mathrm{B}$ subunit of the coat protein dimer by $\sim 0.3 \AA$ compared to the F5 complex structure. This phosphate has been shown to have an important role in stem-loop affinity (Johansson et al. 1998), consistent with its numerous hydrogen bond interactions with coat protein. It is possible that its closer proximity to the $\mathrm{B}$ subunit also contributes to the increased affinity since several hydrogen bonds are shortened by this movement (Fig. 4).

A number of protein-RNA hydrogen bond interactions are longer in the F5/2AP10 complex compared to the F5 complex (Fig. 4). Increases in protein-RNA bond lengths for Asn B55 to the -7 phosphate and Lys B61 to the -10 phosphate both occur as a result of slight changes in the relative phosphate atom positions. Similarly, there is an increase in the intramolecular bond length of one of the two hydrogen bonds between the 2 ' hydroxyl of $\mathrm{G}-11$ and the -9 phosphate; this also occurring due to a change in the position of the -9 phosphate. The only other increased hydrogen bond length of note is that between the O $\gamma$ of Thr B45 and the N3 of the 2AP base at the -10 position. These changes are correlated with the rotation of the $2 \mathrm{AP}$ base within the -10 binding pocket. Several hydrogen bond interactions observed in the F5 complex are not seen in the F5/2AP10 complex, most notably one of the two between the -9 phosphate and the $2^{\prime}$ hydroxyl of G -11 (Fig. 4 ). The position of the -8 phosphate is also changed slightly, leading to the abolition of the hydrogen bond to Arg B49. Both of these changes are also the results of the small backbone movements. We modeled 159 water molecules into the structure.
A

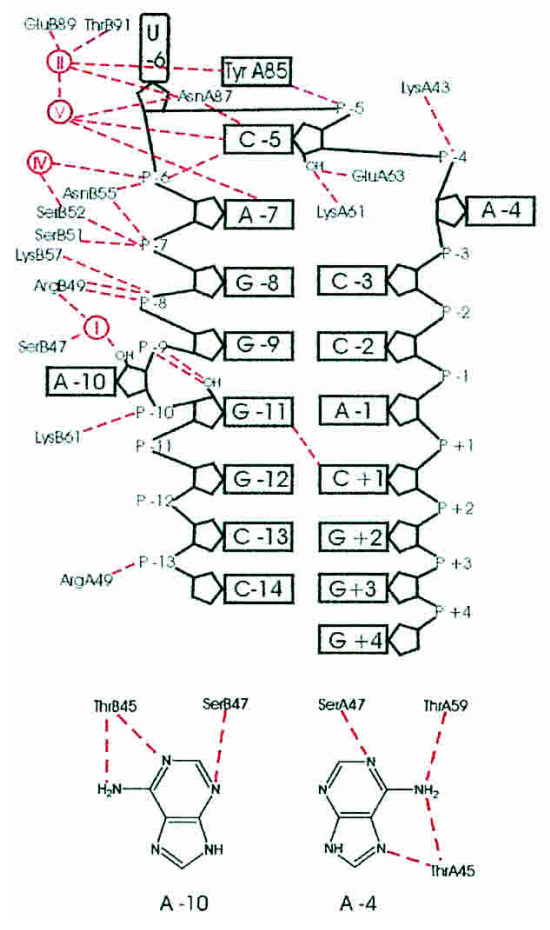

B

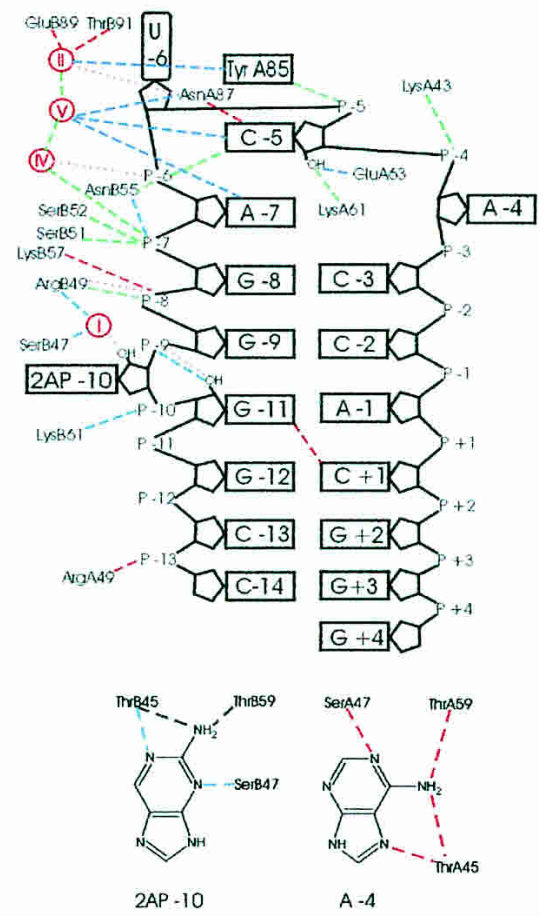

FIGURE 4. Schematic diagram of the hydrogen bond interactions for the F5 $(A)$ (Rowsell et al. 1998) and F5/2AP10 complexes (B), where atoms $3.3 \AA$ or less apart are considered to be within hydrogen bonding range. Ordered water molecules (numbered I-IV) involved in protein-RNA interactions are shown as red circles. Hydrogen bond contacts are represented in $B$ as dashed or dotted lines to highlight differences between the structures and are color coded as follows: red, same length as in $A$; green, shorter than in $A$; blue, longer than in $A$; pink, present only in $A$; and black, present only in $B$. Hydrogen bond contacts are considered to be of lengths different than those of the F5 structure if they differ by $\pm 0.2 \AA$ in the F5/2AP10 structure. 


\section{The F5/2AP10 complex: Implications for modeling and probing ligand-RNA interactions}

The structural determination of the F5/2AP10 RNA complexed with MS2 coat protein has identified a plausible explanation for the high affinity of this variant in solution (Parrott et al. 2000). The increased affinity (approximately threefold greater than its parental F5 sequence) can be rationalized primarily by the formation of a hydrogen bond between the exocyclic amino group of the 2AP base at the -10 position and the backbone carbonyl of Thr B59. A threefold increase in affinity is easily accounted for by an additional intermolecular hydrogen bond (Fersht et al. 1986). The largest factor in determining which of the other RNAs that fulfill the requirements of the consensus sequence have highest affinity is the presence of the $\mathrm{C}$ at the -5 position. The exocyclic amino group of this base points directly at the phosphate at position -6 in the complex, presumably making a favorable electrostatic interaction. Conversely, the $\mathrm{O} 4$ of uridine or modified bases in which the electronegativity of the $\mathrm{O} 4$ position of a pyrimidine at -5 is increased all have significantly lower affinity $(>20$ fold) (Grahn et al. 2000, 2001). These data are all consistent with the 4-amino group helping to order the RNA stemloop into a conformer closer to that recognized by the coat protein. The F5/2AP10 variant benefits from both these factors, hence its overall affinity.

The results presented here and elsewhere on the MS2 system have a number of general implications for studies of RNA-protein interactions. First, the technique of mapping important interactions in RNAs by using chemically variant nucleotides, introduced either by chemical synthesis (Talbot et al. 1990) or transcription (Cochrane et al. 2003), must be used with care. Loss of an interaction that leads to an observable reduction in affinity correlates well with structural studies. However, unchanged or even increased affinities do not necessarily imply that the modified functional group is not an important feature in the complex being probed.

Second, the current MS2 database of over 20 RNA-protein complexes, encompassing variants in RNA or protein sequences, and in RNA functional groups, identify very clearly some of the challenges to be overcome in de novo design of RNA-binding ligands. In principle, molecular modeling could have predicted the formation of the additional intermolecular hydrogen bond seen in the F5/2AP10 complex. It is unlikely, however, to have identified the subtler changes in the RNA-protein interface, such as the small rotation of the 2AP base. This rotation in turn is facilitated by the inherent flexibility of the phosphodiester backbone, resulting in movements of the phosphate atoms at the -7 , $-8,-9$, and -10 positions. In most cases the movements result in only small changes to hydrogen bond lengths (Fig. 4). The single exception is the loss of the hydrogen bond between the -9 phosphate and the $2^{\prime}$-hydroxyl of the -10 ribose that is present in the F5 complex (Rowsell et al.
1998). It is difficult to see how such a constellation of subtle conformational changes could be modeled accurately, despite the wealth of structural knowledge in this system.

The F5/2AP10 complex structure adds new weight to a hypothesis tentatively implied by earlier crystallographic studies (Valegård et al. 1997; Convery et al. 1998; Rowsell et al. 1998; van den Worm et al. 1998; Grahn et al. 2000, 2001; Helgstrand et al. 2002). These suggest that the torsional flexibility of the RNA sugar-phosphate backbone facilitates the maintenance of the majority of the protein-RNA interactions seen in the wild-type complex (Valegård et al. 1997) despite changes to sequence or functional groups on the RNA.

This feature of the RNA-protein interaction becomes obvious when we compare the known MS2 coat protein-stemloop structures (Fig. 5). Aligning RNA conformations by
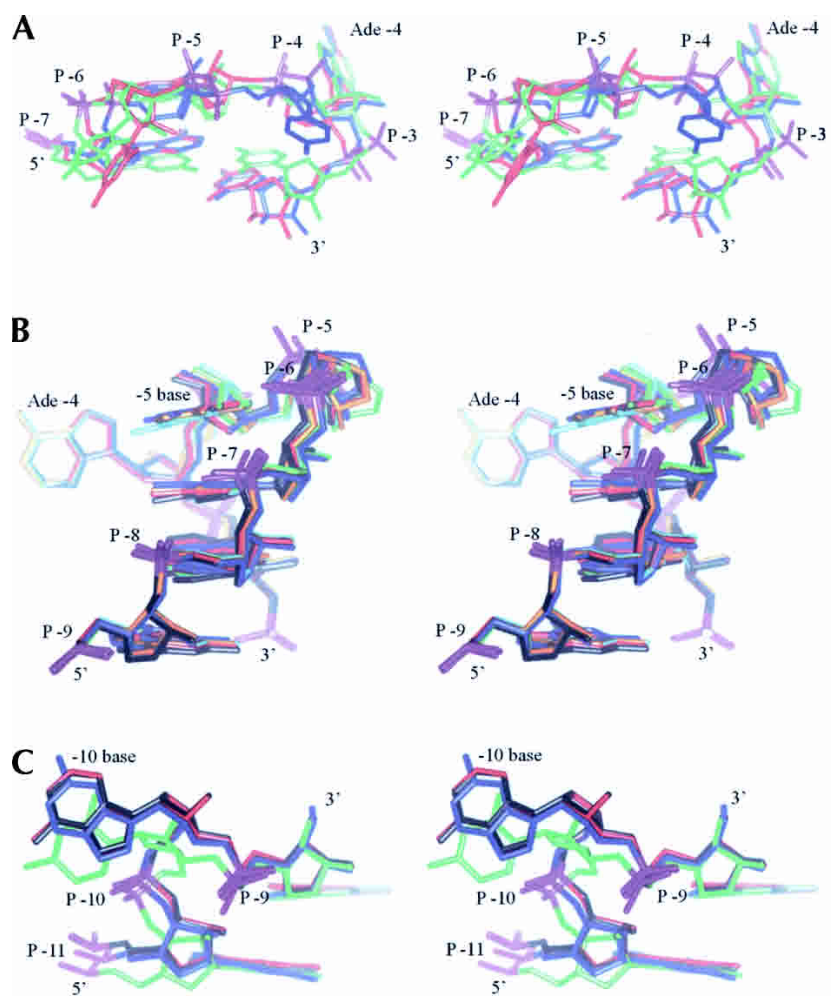

FIGURE 5. Stereo diagrams showing the variation in positions of phosphate groups (shown in purple) for a variety of substituted stemloops whose complexes with wild-type coat protein have been determined. All stem-loops are shown overlaid onto the wild-type structure (PDB code 1ZDI) shown in red, using the coordinates of the coat protein subunits at the $\mathrm{AB}$ position as the reference. $(A, B)$ Differing views of base variants in the loop region, oriented to emphasize changes in phosphate positions. (C) Similar superposition for the available -10 base variants. Color codes for the individual RNA structures are as follows with their PDB codes in parentheses. (A) F6 aptamer, green (6MSF); TR/ -5 4one variant, blue (1ZDS). (B) TR/-5 4-thiouridine, green (1HEO); TR/-5 5BrU, dark blue (1E6T); TR/-5 G, light blue (1H8J); TR/-5 A, orange (1HE6); TR/-5 C, black (1ZDH). U-6 base omitted for clarity. (C) TR/ $-5 \mathrm{C}$, black (1ZDH); F5/2AP10, blue and TR/-10 C, green (1KUO). Figure created using the program SPOCK. 
using protein coordinates as a reference reveals a number of clear conformational themes. In general, there is more conformational rearrangement of the phosphodiester backbone in the region of the substitution. We have many more examples of variants in the loop region than at position -10 , but in both regions there is considerable backbone flexibility. In the loop region the phosphate groups move by $0.4-$ $2.0 \AA$, whereas at the -10 position movements by up to 0.6 $\AA$ are observed.

The formation of hydrogen bonds between the phosphate backbone and the protein has been shown to be an important determinant of binding affinity (Dertinger et al. 2000; Dertinger and Uhlenbeck 2001). In most of the cases where we have structural information, movement of phosphate atoms does not disrupt such hydrogen bonding, although the bond lengths are altered, presumably with implications for binding free energy. Phosphate backbone flexibility is therefore a major determinant of RNA-ligand affinity and must be incorporated into approaches toward ligand design.

\section{MATERIALS AND METHODS}

\section{Expression, crystallization, and RNA synthesis}

MS2 coat protein was overexpressed in E. coli and purified using previously described methods (Mastico et al. 1993). Crystals of MS2 capsids, free of native RNA, were grown using previously described crystallization conditions (Valegard et al. 1986) and the hanging drop technique of vapor diffusion. Crystals were up to 1.5 $\mathrm{mm}$ (largest dimension) in size, having space group R32 with cell dimensions $a=b=288.0 \AA$ and $c=653.0 \AA$. The F5 aptamer RNA stem-loop with $2 \mathrm{AP}$ substituted at the -10 position was synthesized via solid phase phosphoramidite chemistry (Murray et al. 1994), the modified base precursor (5'-O-(dimethoxytrityl)-N-2(dimethyl-aminomethylidene)deoxypurineriboside-3' -O-((2-cyanoethyl)- $N, N$-isopropyl)-phosphoramidite) being purchased from Glen Research. The crystals were soaked with the F5/2AP10 RNA, at a final concentration of $2 \mathrm{mg} / \mathrm{mL}$, for $5 \mathrm{~d}$ prior to mounting in glass capillaries for data collection.

\section{Data collection and processing}

Diffraction data were collected at beamline 14.1 (SRS), with wavelength $1.244 \AA$ and oscillation angle $0.5^{\circ}$. Data were collected at $25^{\circ} \mathrm{C}$ using an ADSC Quantum 4R CCD detector. Data were processed using MOSFLM (Collaborative Computational Project Number 4 [CCP4] 1994) and scaled using SCALA (CCP4 1994). The program TRUNCATE (CCP4 1994) was used to derive structure factor amplitudes from the scaled data. Statistics from the data collection and scaling are shown in Table 1.

\section{Structural determination and refinement}

Initial phases for the data were derived using the published coordinates of the MS2 capsid (PDB entry 2MS2; Golmohammadi et al. 1993) as a phasing model. The X-PLOR program suite (Brünger et al. 1987) was utilized for the refinement of the structure with stereochemical restraints applied throughout the refinement for both the protein (Engh and Huber 1991) and the nucleic acid (Parkinson et al. 1996). Restraints for the 2AP base were calculated using the PRODRG server (van Aalten et al. 1996). An initial round of rigid body refinement (40 minimization steps) was applied to the capsid crystallographic asymmetric unit, $2 F o-F c$ and $F o-F c$ electron density maps were calculated, and real space averaging over the 10-fold noncrystallographic symmetry was carried out using the RAVE package. Despite the incompleteness of the data, the quality of the electron density maps was excellent because of the application of the 10-fold noncrystallographic symmetry averaging. Refinement of atomic positions (80 cycles per refinement round) was alternated with the refinement of the individual atomic temperature factors ( 20 cycles per refinement). Strict noncrystallographic symmetry, with all subunits of the same type constrained to be identical, and a bulk-solvent correction were applied throughout the refinement.

A molecular envelope for the icosahedral asymmetric unit, containing the three protein capsid subunits $\mathrm{A}, \mathrm{B}$, and $\mathrm{C}$ and the regions corresponding to the bound RNA, was generated using the coordinates of the wild-type complex (PDB entry 1ZDI; Valegard et al. 1997). Protein atoms were given an envelope radius of $2 \AA$ and nucleic acid atoms a radius of $5 \AA$ to allow for potential differences in RNA structure prior to applying noncrystallographic symmetry. Envelopes were calculated using the program MAMA within the RAVE package (Kleywegt and Jones 1994). The electron density for the bound RNA was clearly defined and the RNA at the $\mathrm{AB}$ and $\mathrm{CC}$ binding sites was modeled using the program $\mathrm{O}$ (Jones et al. 1991).

After both the AB and CC RNA had been built into the model, water molecules were added using the Watpekpik routine in " $O$ " (Jones et al. 1991). The occupancy of the RNA at the AB site was chosen so that the adjacent protein and RNA atoms had similar temperature factors. A summary of the refinement statistics for the complex is given in Table 2.

Analysis of the stereochemistry of the final model was carried out using PROCHECK (Laskowski et al. 1993; CCP4 1994). The Ramachandran plot showed that there were only two residues in disallowed or generously allowed regions. Both residues (Asn C36 and Ser B2) have been previously observed to have unusual backbone angles in several other capsid-RNA stem-loop complexes (Valegård et al. 1997; Grahn et al. 2000). The RNA geometry of the complex was checked by calculating the torsion angles for all residues using the program CURVES (Lavery and Sklenar 1988) and comparing the results to the corresponding values for the $2.5-\AA$ tRNA $^{\text {Phe }}$ structure (Holbrook et al. 1978). All residues displayed torsion angles similar to those observed in the tRNA. The atomic coordinates for the F5/2AP10 complex have been deposited at the Protein Data Bank (PDB) with identification code $1 \mathrm{U} 1 \mathrm{Y}$.

\section{ACKNOWLEDGMENTS}

This work was supported by grants from The Leverhulme Trust, the BBSRC, and MRC.

Received April 27, 2004; accepted August 16, 2004. 


\section{REFERENCES}

Borer, P.N., Lin, Y., Wang, S., Roggenbuck, M.W., Gott, J.M., Uhlenbeck, O.C., and Pelezer, I. 1995. Proton NMR and structural features of a 24-nucleotide RNA hairpin. Biochemistry 34: 64886503.

Brünger, A.T., Kuriyan, J., and Karplus, M. 1987. Crystallographic R factor refinement by molecular dynamics. Science 235: 458-460.

Cochrane, J.C., Batey, R.T., and Strobel, S.A. 2003. Quantitation of free energy profiles in RNA-ligand interactions by nucleotide analog interference mapping. RNA 9: 1282-1289.

Collaborative Computational Project Number 4 (CCP4). 1994. The CCP4 Suite: Programs for protein crystallography. Acta Crystallogr. D 50: 760-763.

Convery, M.A., Rowsell, S., Stonehouse, N.J., Ellington, A.D., Hirao, I., Murray, J.B., Peabody, D.S., Phillips, S.E.V., and Stockley, P.G. 1998. Crystal structure of an RNA aptamer-protein complex at 2.8 Å resolution. Nat. Struct. Biol. 5: 133-139.

Dertinger, D. and Uhlenbeck, O.C. 2001. Evaluation of methylphosphonates as analogues for detecting phosphate contacts in RNAprotein complexes. RNA 7: 622-631.

Dertinger, D., Behlen, L.S., and Uhlenbeck, O.C. 2000. Using phosphorothioate substituted RNA to investigate the thermodynamic role of phosphates in a sequence specific RNA-protein complex. Biochemistry 39: 55-63.

Engh, R.A. and Huber, R. 1991. Accurate bond and angle parameters for X-ray protein structure refinement. Acta Crystallogr. A 47: 392400.

Fersht, A.R., Leatherbarrow, R.J, and Wells, T.N.C. 1986. Structure and activity of the tyrosyl-transfer RNA-synthetase-The hydrogen bond in catalysis and specificity. Philos. Transact. Ser. A Math. Phys. Eng. Sci. 317: 305-320.

Golmohammadi, R., Valegård, K., Fridborg, K., and Liljas, L. 1993. The refined structure of bacteriophage MS2 at $2.8 \AA$ resolution. J. Mol. Biol. 234: 620-639.

Grahn, E., Stonehouse, N.J., Adams, C.J., Fridborg, K., Beigelman, L., Matulic-Adamic, J., Warriner, S.L., Stockley, P.G., and Liljas, L. 2000. Deletion of a single hydrogen bonding atom from the MS2 RNA operator leads to dramatic rearrangements at the RNA-coat protein interface. Nucleic Acids Res. 28: 4611-4616.

Grahn, E., Moss, T., Helgstrand, C., Fridborg, K., Sundarum, M., Tars, K., Lago, H., Stonehouse, N.J., Davis, D.R., Stockley, P.G., et al. 2001. Structural basis of pyrimidine specificity in the MS2 RNA hairpin-coat-protein complex. RNA 7: 1616-1627.

Helgstrand, C., Grahn, E., Moss, T., Stonehouse, N.J., Tars, K., Stockley, P.G., and Liljas, L. 2002. Structures of complexes between MS2 coat protein and RNA translational operator fragments with substitutions at the -10 and -7 positions. Nucleic Acids Res. 30: 26782685.

Hirao, I., Peabody, D., and Ellington, A.D. 1998. The limits of specificity: An experimental analysis with RNA aptamers to MS2 coat protein variants. Mol. Divers. 4: 75-89.

Holbrook, S.R., Sussman, J.L., Warrant, R.W., and Kim, S.H. 1978. Crystal structure of yeast phenylalanine transfer RNA II. Structural features and functional implications. J. Mol. Biol. 123: 631-660.

Johansson, H.E., Dertinger, D., LeCuyer, K.A., Behlen, L.S., Greef, C.H., and Uhlenbeck, O.C. 1998. A thermodynamic analysis of the sequence specific binding of RNA by bacteriophage MS2 coat protein. Proc. Natl. Acad. Sci. 95: 9244-9249.

Jones, T.A., Zou, J.Y., Cowen, S.W., and Kjeldgaard, M. 1991. Improved methods for building models in electron density maps and locations of errors in these models. Acta Crystallogr. A 47: 110119.

Kleywegt, G.J. and Jones, T.A. 1994. Halloween. . .masks and bones. In
From first map to final model (eds. S. Bailey et al.), pp. 59-66. SERC Daresbury Laboratory, Warnington, England.

- 1997. Detecting folding motifs and similarities in protein structures. Methods Enzymol. 277: 525-545.

Laskowski, R.A., Macarthur, M.W., Moss, D.S., and Thornton, J.M. 1993. PROCHECK-A program to check the stereochemical quality of protein structures. J. Appl. Crystallogr. 26: 283-291.

Lavery, R. and Sklenar, H. 1988. The definition of generalised helicoidal parameters and of axis curvature for irregular nucleic acids. $J$. Biomol. Struct. Dyn. 6: 63-91.

Ling, C.M., Hung, P.P., and Overby, L.R. 1970. Independent assembly of Q $\beta$ and MS2 phages in doubly infected E. coli. Virology 40: 920929.

Mastico, R.A., Talbot, S.J., and Stockley, P.G. 1993. Multiple presentation of foreign peptides on the surface of an RNA-free spherical bacteriophage capsid. J. Gen. Virol. 74: 541-548.

Murray, J.B., Collier, A.K., and Arnold, J.R.P. 1994. A general purification procedure for chemically synthesised oligoribonucleotides. Anal. Biochem. 218: 177-184.

Parkinson, G., Vojtechovsky, J., Clowney, L., Brügner, A.T., and Berman, H.M. 1996. New parameters for the refinement of nucleicacid containing structures. Acta Crystallogr. D 52: 57-64.

Parrott, A.M., Lago, H., Adams, C.J., Ashcroft, A.E., Stonehouse, N.J., and Stockley, P.G. 2000. RNA aptamers for the MS2 bacteriophage coat protein and the wild-type RNA operator have similar solution behaviour. Nucleic Acids Res. 28: 489-497.

Rowsell, S., Stonehouse, N.J., Convery, M.A., Adams, C.J., Ellington, A.D., Hirao, I., Peabody, D.S., Stockley, P.G., and Phillips, S.E.V. 1998. Crystal structures of a series of RNA aptamers complexed to the same protein target. Nat. Struct. Biol. 5: 970-975.

Stockley, P.G., Stonehouse, N.J., and Valegård, K. 1994. Molecular mechanism of RNA phage morphogenesis. Int. J. Biochem. 26: 1249-1260.

Stockley, P.G., Stonehouse, N.J., Murray, J.B., Goodman, S.T.S., Talbot, S.J., Adams, C.J., Liljas, L., and Valegård, K. 1995. Probing sequence specific RNA recognition by the bacteriophage MS2 coat protein. Nucleic Acids Res. 23: 2512-2518.

Talbot, S.J., Goodman, S., Bates, S.R.E., Fishwick, C.W.G., and Stockley, P.G. 1990. Use of synthetic oligoribonucleotides to probe RNA-protein interactions in the MS2 translational operator complex. Nucleic Acids Res. 18: 3521-3528.

Valegård, K., Unge, T., Montelius, I., and Strandberg, B. 1986. Purification, crystallisation and preliminary X-ray data of the bacteriophage MS2. J. Mol. Biol. 190: 587-591.

Valegård, K., Murray, J.B., Stockley, P.G., Stonehouse, N.J., and Liljas, L. 1994. Crystal structure of a bacteriophage RNA coat protein operator system. Nature 371: 623-626.

Valegård, K., Murray, J.B., Stonehouse, N.J., van den Worm, S., Stockley, P.G., and Liljas, L. 1997. The three-dimensional structures of two complexes between recombinant MS2 capsids and RNA operator fragments reveal sequence specific protein-RNA interactions. J. Mol. Biol. 270: 724-738.

van Aalten, D.M.F., Bywater, R., Findlay, J.B.C., Hendlich, M., Hooft, R.W.W., and Vriend, G. 1996. PRODRG, a program for generating molecular topologies and unique molecular descriptors from coordinates of small molecules. J. Comput. Aided Mol. Des. 10: 255262.

van den Worm, S.H.E., Stonehouse, N.J., Valegård, K., Murray, J.B., Walton, C., Fridborg, K., Stockley, and P.G., Liljas, L. 1998. Crystal structures of MS2 coat protein mutants in complex with wild type RNA operator fragments. Nucleic Acids Res. 26: 1345-1351.

Witherell, G.W., Gott, J.M., and Uhlenbeck, O.C. 1991. Specific interaction between RNA phage coat proteins and RNA. Progr. Nucleic Acid Res. Mol. Biol. 40: 185-220. 

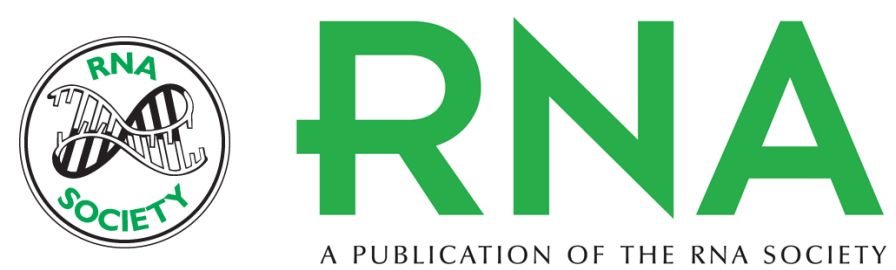

\section{The crystal structure of a high affinity RNA stem-loop complexed with the bacteriophage MS2 capsid: Further challenges in the modeling of ligand-RNA interactions}

WILF T. HORN, MÁIRE A. CONVERY, NICOLA J. STONEHOUSE, et al.

RNA 2004 10: 1776-1782

References This article cites 34 articles, 5 of which can be accessed free at:

http://rnajournal.cshlp.org/content/10/11/1776.full.html\#ref-list-1

License

Email Alerting Receive free email alerts when new articles cite this article - sign up in the box at the

Service top right corner of the article or click here.

To subscribe to RNA go to:

http://rnajournal.cshlp.org/subscriptions 